\title{
Nanotheranostics
}

2017; 1(3): 261-271. doi: 10.7150/ntno.18964

Research Paper

\section{The biocompatibility studies of polymer dots on pregnant mice and fetuses}

\author{
Na Wu1, Zheng Zhang1, Jie Zhou1, Zezhou Sun', Yueyue Deng1, Guimiao Lin², Ming Ying3, Xiaomei \\ Wang ${ }^{2}$, Ken-Tye Yong ${ }^{6}$, Changfeng $\mathrm{Wu}^{4}{ }^{\bowtie}$, Gaixia Xu${ }^{1 凶}$ \\ 1. Key laboratory of Optoelectronic Devices and System of The Ministry of Education/Guangdong Province, College of Optoelectronic Engineering, Shenzhen \\ University, Shenzhen Guangdong Province 518060, China; \\ 2. Key laboratory of Biomedical Engineering of Shenzhen, College of medicine, Shenzhen University, Shenzhen, Guangdong 518060, China; \\ 3. Shenzhen Key Laboratory of Marine Bioresource and Eco-environmental Science, Shenzhen Key Laboratory of Microbia Genetic Engineering, College of \\ Life Sciences and Oceanography, Shenzhen University, Shenzhen 518060, China; \\ 4. Department of Biomedical Engineering, Southern University of Science and Technology, Shenzhen, Guangdong 518055, China; \\ 5. State key laboratory on integrated Optoelectronics, College of Electronic Science and Engineering, Jilin University Changchun, Jinlin 130012, China; \\ 6. School of Electrical and Electronic Engineering, Nanyang Technological University, Singapore 639798, Singapore. \\ $\bowtie$ Corresponding authors: xugaixia@szu.edu.cn, wucf@sustc.edu.cn
}

(c) Ivyspring International Publisher. This is an open access article distributed under the terms of the Creative Commons Attribution (CC BY-NC) license (https://creativecommons.org/licenses/by-nc/4.0/). See http://ivyspring.com/terms for full terms and conditions.

Received: 2016.12.28; Accepted: 2017.05.02; Published: 2017.06.09

\begin{abstract}
Semiconducting polymer dots (Pdots) are small nanoparticles consisting primarily of fluorescent pi-conjugated polymers which show superior optical properties for biological imaging and biosensors. It is necessary to explore systematically the toxicity of Pdots on animals before extensive biomedical applications. The reproductive system is very sensitive to the external invasion and essential for species reproduction as well. In this work, we used the pregnant mice to investigate the reproductive toxicity of Pdots. The changes in body weight of each maternal mouse were recorded every two days. The main organs were collected and analyzed as soon as all the pregnant mice were sacrificed on the $15^{\text {th }}$ embryonic day. Distributions of Pdots on maternal major organs and tissues were examined in frozen tissue sections. Hematoxylin and eosin (H\&E) staining was performed to investigate the histopathological changes of maternal organs. The blood chemistry test was applied to study the effects of Pdots on organ functions. Female hormones were evaluated by immunoassays. The amniotic fluid was inspected for assessing their penetration ability of Pdots. Levels of placenta growth related factors were detected by RT-PCR to evaluate the function of placenta. These results showed that Pdots were mainly accumulated in liver and spleen, and no apparent impact was observed on maternal body weight and organs coefficients. Histopathological images also showed normal tissue morphology compared with the untreated group. The female hormones levels did not show significant difference among the three groups as well. Trace amount of Pdots could get into the amniotic fluid but did not change the placental functions and the early development of fetus. Our results demonstrated that Pdots have excellent biocompatibility and no reproductive toxicity under the dosages used in this work, which means that Pdots have great potential in preclinical applications in the future.
\end{abstract}

Key words: nanoparticles, polymer dot, reproductive toxicity.

\section{Introduction}

Quantum dots (QDs), known as semiconductor nanocrystals, have made a tremendous impact on biomedical area owing to their superior physical optical properties such as high photo-stability, narrow and symmetric emission spectrum and high fluorescent quantum yield [1]. During the past decades, traditional QDs such as Cd-containing QDs have been used as highly sensitive fluorescent probes for in vitro labeling [2], single molecule tracking [3] as well as in vivo tumor targeting and drug delivering 
[4]. However, although there are wide applications of quantum dots, many potential hazards of QDs still raised various concerns for us. It is reported that Cd-containing QDs could be taken up through endocytosis and degraded to release cadmium ions, which augments their toxicity apparently [5]. Toxicities of Cd-containing QDs including immune toxicity, neurotoxicity and reproductive toxicity have been extensively studied in different in vitro and in vivo models [6, 7]. Li et al. found that CdTe QDs could be accumulated in male mouse testis and dose-dependently damaged the male mice's reproductive system such as the damaging testis tissue structure, disrupting hormone levels and decreasing sperm quality [8]. Similarly, Chu et al. found that $\mathrm{CdTe} / \mathrm{CdS}$ could cross through the placenta barrier in pregnant mice and distribute in the tissue of fetus, which increased the death rate of the fetal [9]. Besides, Cd-containing QDs could suppress the spleen-derived lymphocyte immune functions of BalB/c mouse too [10]. Therefore, it is essential to develop fluorescence nanoparticle with low potential in toxicity.

Recently, polymer dots have attracted attention great deal of interest as novel fluorescence nanoparticles because they do not contain heavy metals. The polymer dots are mainly composed of $\Pi$-conjugated polymers which are wide-band-gap semiconductors in their pristine states. Furthermore, the direct band gap structure of this semiconductor polymer makes it absorb and emit effectively on the band edge. Previous studies indicated that polymer dots have shown multicolor emissions by changing the composition of different hydrophobic polymers [11]. In previous work, we have reported on several types of polymer dots which exhibited high fluorescence brightness as compared with fluorescent dyes and inorganic QDs [12]. The brightness of PF-TC6FQ polymer nanoparticles designed by Liu's group was around 8 times brighter than QDs655/QDs705 under the $488 \mathrm{~nm}$ excitation [13]. All these good characteristics enabled polymer dots to be a potential for nanoparticles in biomedical applications. Liu et al. had successfully traced the in vitro cell and monitored the in vivo liver tumor growth by utilizing the near-infrared conjugated polymer dots $[14,15]$. We have already used polymer dot in cellular imaging and obtained clear cellular morphology and structure [16].

Although the polymer dots have been used in biomedical imaging and biosensors, the toxicity of Pdots on living animals is still rarely explored. Because the reproductive system of mammal is very sensitive in protecting embryo from external invasion [17], it is an ideal model to detect the in vivo toxic effects of nanoparticles. Here we established a reproductive model to investigate the toxic effects of Pdots. The pregnant mice were injected Pdots every two days from embryonic day 0 (ED0). After the injections, the pregnant mice were sacrificed on ED15. Finally, the organ index, reproductive parameters of pregnant mice (maternal body weight change, organs coefficients, bio-distribution of Pdots, histopathology of tissues and female hormones levels), fetus related index, placenta index and existence of Pdots in amniotic fluid on offspring level were detected and analyzed.

\section{Materials and methods}

\section{Preparation and characterization of Pdots}

The Pdots were prepared by utilizing a visible light-harvesting polymer poly[(9,9-dioctyfluorenyl2,7-dily)-co-(1,4-benzo- $\left\{2,1^{\prime}, 3\right\}$-thiadazole)](PFBT) as the donor, an efficient deep-red emitting polymer poly(9,9-dioctylfluorene)-co-(4,7-di-2-thienyl-2,1,3-be nzothiadiazole) (PF-5DTBT) as the acceptor, and a carboxyl functional polymer PSMA [poly(styrene-co-maleic anhydride)]. Both the PFBT and PF-5DTBT were purchased from ADS Dyes, Inc. (Quebec, Canada). PSMA as well as other reagents for Pdots preparation were purchased from Sigma-Aldrich (St. Louis, MO, USA). Firstly, PFBT, PF-BDT5 and PSMA were respectively dissolved into tetrahydrofuran (THF) and mixed at a ratio of 5:3:2 as the precursor solution. Then we obtained the Pdots in a similar method as aforementioned [11]. In this study, Pdots solution was diluted in PBS for further use. For sample characterization, a Nano-particle Size Analyzer (Malvern Nano-ZS90, British) was used to get Hydrodynamic diameter Distribution and Zeta potential. The absorbance and emission spectrum of Pdots were measured by fluorescence spectrometer (Hitachi FL-4600, Japan) with quartz cuvettes. Morphology of Pdots was analyzed by Transmission electron microscopy (TEM) (JEM-1230, JEOL). All these characterizations were kept stable at room temperature for several months.

\section{Reproduction model construction}

8 to 9 weeks old female and male Kunming mice (KM) were purchased from Experimental Animal Center of Guangdong Province, China (SCXK2013-0002). Female and male mice were put in cages in the proportion of 2:1. The day when the female mouse has virginal plug was defined as embryonic day 0 (ED0). After getting pregnant, three pregnant female mice were kept in a cage, fed with the same routine and housed in the same animal room in a 12 hours light and dark cycle during their pregnancy. Referring to our previous work about 
bioimaging and reproductive toxicity, we divided the KM pregnant mice into three groups $[12,18]$. Two Pdots treated groups and one blank group. $100 \mu \mathrm{L}$ PBS solution including $25.0 \mu \mathrm{g}$ and $12.5 \mu \mathrm{g}$ Pdots respectively were injected into each pregnant mouse through the tail vein every two days for 7 times (175.0 $\mu \mathrm{g}, 87.5 \mu \mathrm{g}$ Pdots respectively in total) in the first two pregnant weeks. At the same time, $100 \mu \mathrm{L}$ PBS solution was injected into each pregnant mouse in untreated the group for 7 times. Animal handling during the experiment was carried out according to the standard animal practice and regulation of Shenzhen University Laboratory Animal Center. All the animal maintenance and experiments were approved by the Laboratory Animal Ethics Committee of Shenzhen University (AEWC201501003).

\section{Weight measurement and sample collection}

After getting pregnancy, all the weights of the female mice were recorded at the same time before injection every two days from the ED0 to ED14. After blood from the mouse's eyeballs was taken, each pregnant mouse was sacrificed by neck isolation on ED15. Major organs (heart, liver, spleen, lung, kidney, brain and ovary) of mice were harvested as soon as possible and then their weights were recorded. Finally, we calculated the maternal weight change and organs coefficient. (Organs coefficient=organs weight / body weight)

\section{Frozen section and histological measurement}

For investigating the distribution of Pdots in organs, some of the main maternal organs were frozen, and then were sectioned. Firstly, we put some optimal cutting temperature compound (OCT) embedding medium to embed the organs respectively. Then, we sectioned the organs into 7 microns slices by freezing microtome (CM1850, LEICA). Each slice was adhered on the glass slide. Finally, we observed the distribution of Pdots under the fluorescence microscope (BX53, OLYMPUS). The remaining organs' tissues of maternal mouse were preserved in $4 \%$ phosphate buffered formalin at $4^{\circ} \mathrm{C}$ for more than 48 hours. After that, the resected tissues were placed in histology cassettes where they were infiltrated and embedded by paraffin with embedding machine (EG1150H, LEICA). Later, we sectioned the tissues by paraffin section machine (RM2235, LEICA). All slices stained by hematoxylin and eosin were examined with a light microscope (BX53, OLYMPUS) according to standard procedures.

\section{Female hormones level and blood biochemistry analysis}

For detecting the levels of maternal female hormones (estrogen and progesterone) and blood chemistry, maternal blood was harvested from each mouse through the eyeballs and collected in centrifuge tube respectively before being sacrificed. The blood tubes were placed at $4{ }^{\circ} \mathrm{C}$ overnight and then we collected their serum. Both the levels of estrogen (E2) and progesterone (PG) in serum were detected respectively by the specific kit of Enzyme-linked immunoassay (ELISA) that was purchased from Cloud-Clone Corp. Besides, the blood biochemistry indicators for liver and spleen, aspartate transaminase (AST), alanine transaminase (ALT), alkaline phosphatase (ALP), albumin (ALB), globulin (GLB), A/G=ALB/GLB, were assayed by Blood Biochemical analyzer (Mindray, BS-200).

\section{Fetotoxicity evaluation}

Embryos including placentas and fetuses were removed and separated immediately after maternal death. All the weights of fetuses were recorded and inspected on whether the fetal facial features and limbs have any abnormalities and deformities. Each fetus weight was recorded for estimating its healthy condition.

\section{Existence of Pdots in amniotic fluid}

The amniotic fluid was collected from the embryo before separating the placenta and fetus. All the amniotic fluid of each maternal mouse was collected into one centrifuge tube and centrifuged at 3000rmp for 5 minutes, the sediment was collected. Later, the sediment was smeared on a glass slide and covered with coverslips. Finally, the Pdots distributions were examined in the sediment with a fluorescence microscope.

\section{The placental barrier function evaluation}

Placental barrier is an important defense to protect the fetus from external invasion. Here, we assessed the transcription level of placental growth factor (PGF) as well as the placenta vascular endothelial growth factors (VEGF) to detect whether the placental barrier function is normal or not. The total RNA of each placenta was gathered with Trizol method. Finally, we used the Real-time PCR to measure the expression level of target genes.

\section{Statistical analysis}

The quantitative data such as maternal body weight change, organs coefficient as well as the female hormones level were presented as means \pm standard deviation $(\mathrm{SD}, \mathrm{n} \geq 6)$. Statistical significance in the difference between groups were evaluated by Student's t-test and $\mathrm{P}<0.05\left(^{*}\right)$ was considered as a statistical significance. 


\section{Results and Discussion}

\section{Pdots preparation and physical characterization}

The properties of the synthesized Pdots used in our study were characterized by TEM, Nano-particle size analyzer and UV-Vis/PL Spectra. As shown in Figure $1 \mathrm{~A}$ and $1 \mathrm{E}$, the TEM image indicated that Pdots had an average size of $20 \mathrm{~nm}$ and mono-dispersed morphology without obvious aggregation. The average hydrodynamic diameter of the Pdots were around $24 \pm 6.88 \mathrm{~nm}$. The deviation in diameter determined by TEM and DLS was attributed to the completely different surface morphology of the particle under different tested conditions [19]. Surface carboxyl groups of this Pdots unfolded in aqueous condition which resulted in the hydrodynamic diameter being a little larger than that derived from TEM image. Figure 1B indicated that Chemical structures of the Pdots. PFBT played as the donor with the absorption peak at around $460 \mathrm{~nm}$ and the
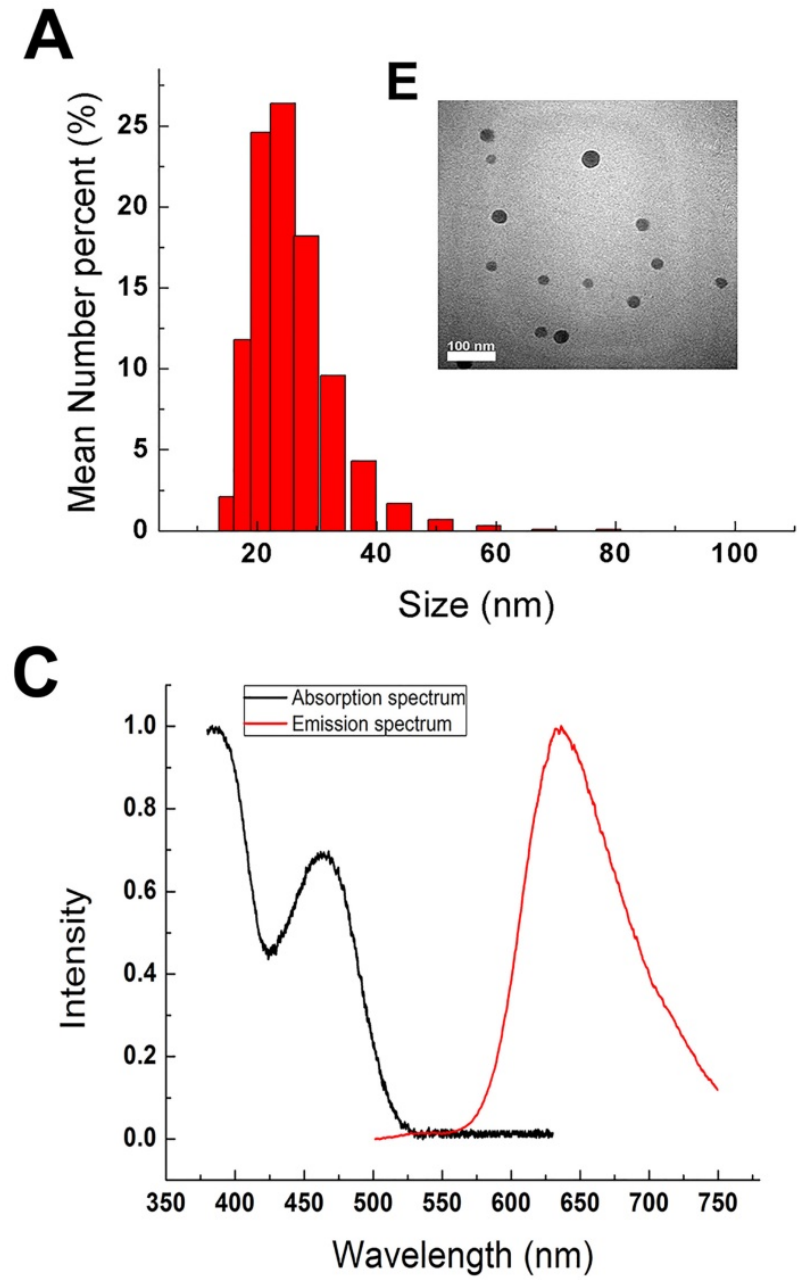

emission peak at around $540 \mathrm{~nm}$, PF-DBT5 played as acceptor with the absorption peak at around $380 \mathrm{~nm}$ and the emission peak at around $635 \mathrm{~nm}$ [11]. Since both the donor and the acceptor had been closely packed into a single dot, the intra-particle energy transfer made the donor completely quenchable and the acceptor fluorescent. Therefore, the absorption spectrum ranged from UV to $530 \mathrm{~nm}$ with the absorption peaks of $380 \mathrm{~nm}$ and $460 \mathrm{~nm}$ (Figure 1C), while the single emission peak of photoluminescence spectra was $635 \mathrm{~nm}$ and the half tall width was around $100 \mathrm{~nm}$. The zeta potential of Pdots was $-35.4 \pm 4.21$ $\mathrm{mV}$, which could avoid the interaction between Pdots and some negatively charged molecules in the blood. Also, the efficient deep-red emission made them approximately 15 times brighter than QD655 [12]. Besides, the biggest difference between Pdots and Cd-containing QDs was that Pdots were mainly composed of polymer material such as $\mathrm{C}, \mathrm{H}, \mathrm{O}, \mathrm{N}$ and $\mathrm{S}$, which avoided the toxicity risk of heavy metal elements completely.

B
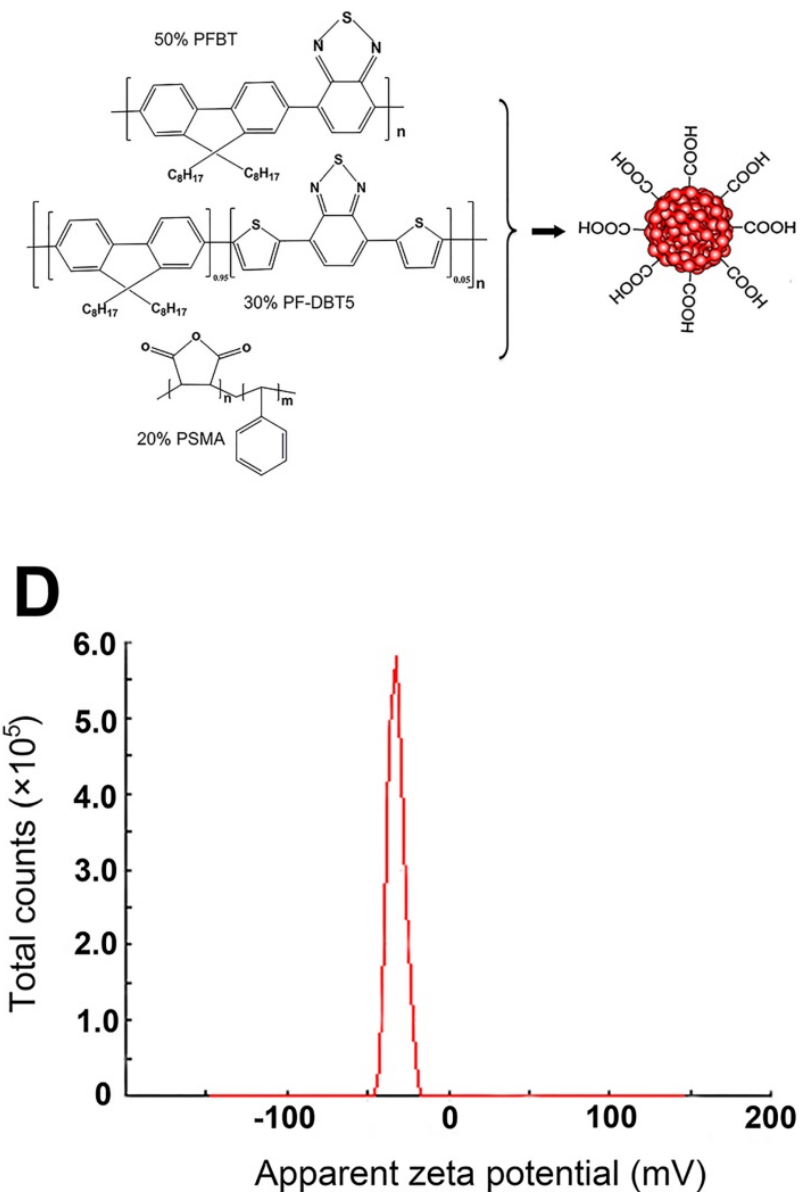

Figure 1. Physical characterizations of Pdots suspended in deionized water. A) The hydrodynamic diameter of Pdots. B) Chemical structures of the Pdots, polymer PFBT and PF-DBT5 were the core fluorescent material while polymer PSMA was a kind of amphiphilic material which offered the carboxyl to the surface of Pdots. C) Absorption and Emission spectra of Pdots. D) Zeta potential of the Pdots. E) The TEM image of Pdots. 


\section{Toxic effect of Pdots on maternal body weight and organ coefficient}

Fluctuations of the body weight and organ weight coefficients were considered as common indicators for evaluating the animal status [20, 21]. In this study, maternal body weights were continuously recorded every two days from the ED0 to ED14 for investigating the toxic effects of Pdots in maternal mice. As shown in Figure 2A, the average maternal weight of control group, $12.5 \mu \mathrm{g}$ treated group and $25.0 \mu$ g treated group, increased $60.19 \pm 0.058 \%, 66.31$ $\pm 0.06 \%$ and $49.70 \pm 0.066 \%$ respectively. No apparent difference could be observed between treated groups and untreated group. Previous study has shown that the same reproductive model treated by CdSe-Zns core-shell structure quantum dots functionalized with amphiphilic polymer shell would obtained the same growth trend and weight gain ratio [22]. Therefore, we assessed that polymer composition or shell of nanoparticles has a better biocompatibility for in vivo studies. The organ coefficients of brain, heart, liver, spleen, lung and kidney shown in Figure 2B, have no significant difference between the treated groups and control group. These results further demonstrated that Pdots were well tolerable in pregnant mice and had shown no obvious effect on the body weight and organ coefficients of pregnant mice under our dosage levels.

\section{Distributions and histology analysis}

In order to study the distribution of Pdots in pregnant mice, their major organs were removed and frozen in slices with $7 \mu \mathrm{m}$ thickness for Pdots fluorescence imaging. In this study, the strongest fluorescence could be identified in liver, as well as in spleen, while lower red fluorescence was observed in lung, only sporadic fluorescence signal of Pdot could be found in kidney and heart, no Pdots were found in brain through detecting its fluorescence signal (Figure 3 ). The further relative quantified information of Pdots distribution in maternal organs and embryonic tissue were shown in Figure S1 and Table S1. It was well known that the bio-distribution of nanoparticles was relevant to the particle size, surface coating, zeta potential and PEGylation as well [23]. QDs without PEGylation were more likely to remain intact in liver and spleen because PEGylation could avoid the uptake of reticuloendothelial system (RES) [24]. Other researches in both rats and mice had confirmed that negatively charged nanoparticles are more prone to gathered in liver and spleen through passive targeting $[25,26]$. Although surface modification of carboxyl could increase Pdots hydrophilic function, it did not reduce the absorption of RES and the effect of liver detoxification system and spleen immune system on its distribution. So it was expected that Pdots were mainly gathered in liver and spleen. Our distribution results also coincided with that of the previous researches on Cd-containing QDs. Su et al. reported that Cd-containing QDs were mostly accumulated in liver within 4 hours after tail vein injection [19], because of the uptake of Kupffer cells, the main composition cell of RES [27].The results revealed that tissue distribution of Pdots mainly depended on their sizes and surface chemistry since that nanoparticle hydrodynamic diameter less than $5.5 \mathrm{~nm}$ would not be excreted through renal system [28], while the nanoparticles size was mainly depended on the nano-structure and surface chemistry.

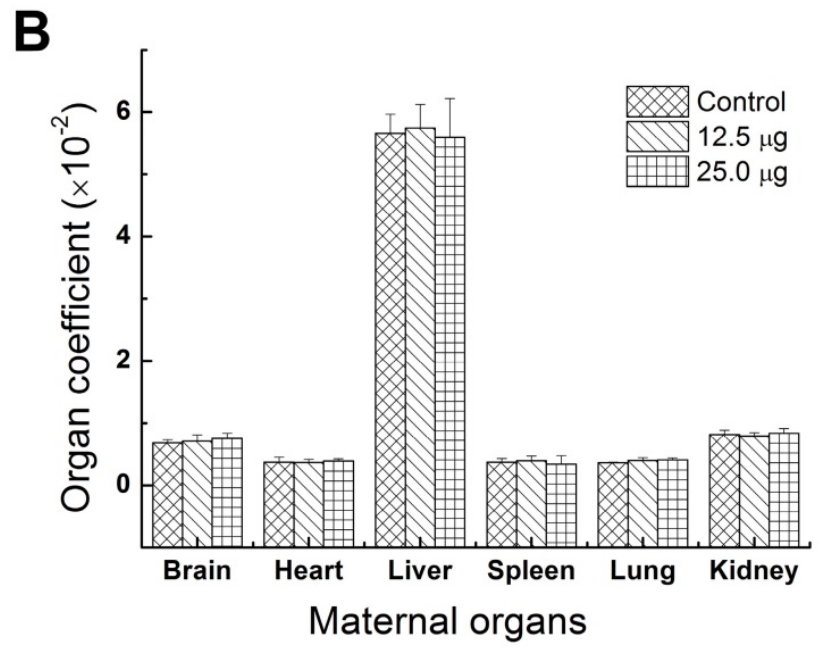

Figure 2. Physical indexes of maternal mice. A) Change in maternal body weight, each maternal mouse was weighted at ED0, ED02, ED04, ED06, ED08, ED010, ED012, ED14 before injection of Pdots, $n=6 . B$ ) Maternal organ index, the main maternal organs including brain, heart, liver, spleen, lung and kidney were harvested from maternal mouse and wet weighted immediately on ED 15, $n=6$ (organ index= wet organ weight/ body weight $\times 100 \%$ ). 


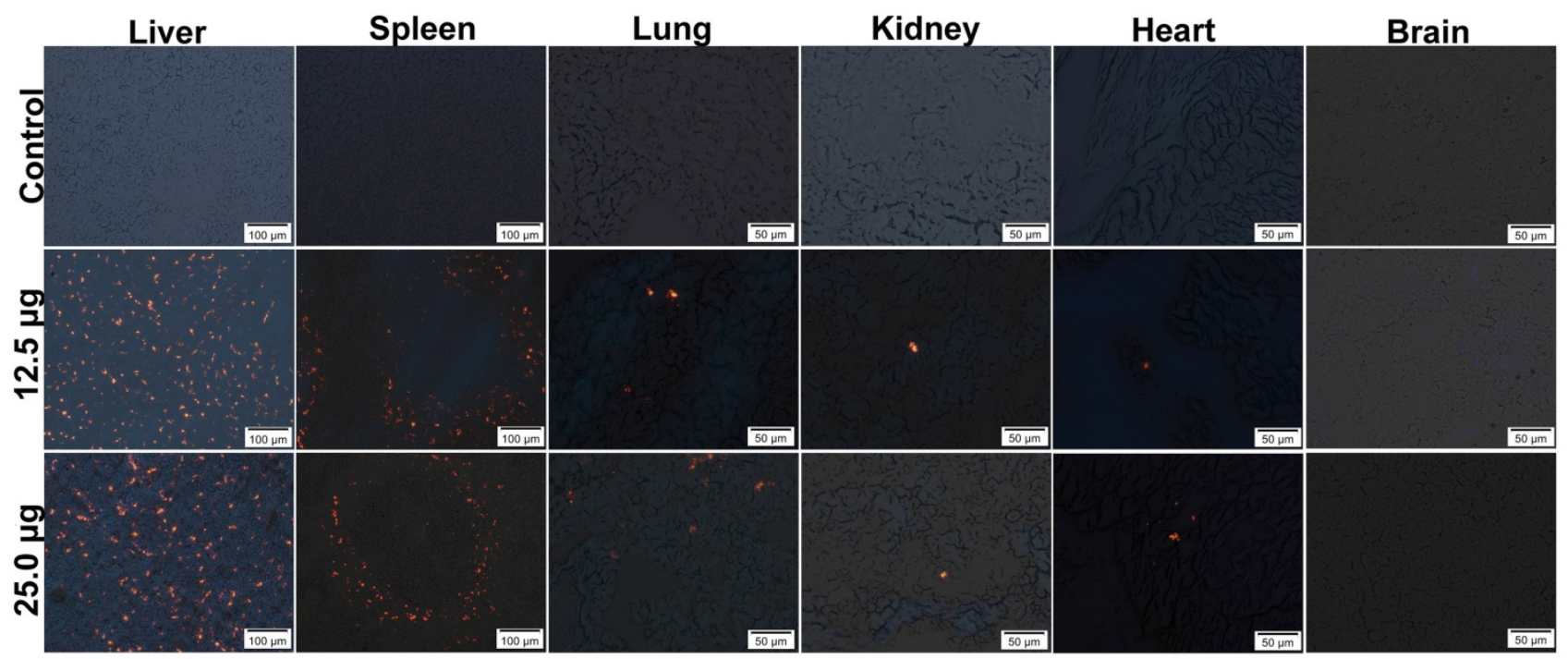

Figure 3. Distributions in maternal main organs (liver, spleen, lung, kidney, heart), the aggregated Pdots emitted orange-red fluorescence under the fluorescence microscope, and Pdots distributed in liver, spleen, lung, kidney as well as heart of maternal mouse among the groups are dose dependently.

The nanotoxicity was caused that the foreign substance was delivered and distributed and degraded in organs in vivo [29]. Since most of the Pdots were mainly distributed in liver and spleen of maternal mouse, it was essential to examine pathological effects of Pdots on these two organs. Further scrutiny was carried out to detect the potential damage of Pdots on maternal tissues. The tissues' slices were stained with H\&E and then observed for the histological differences from treated groups to untreated groups. As shown in Figure 4A, there was no evident histological abnormality between Pdots groups and untreated group. In the aspect of biochemistry analysis, we measured the levels of AST, ALT, ALP, ALB, GLB and A/G, which were sensitive indicator to reflect the function of liver and spleen [29]. As the data shown in Figure 4B and $4 \mathrm{C}$, there were no differences between treated groups and control group. Although Pdots mainly accumulated in these organs, they caused no pathological injuries or inflammation to the tissue structure of liver and spleen demonstrated by our histological blood biochemical analysis. Similarly, Lin et al. reported the InP/ZnS QDs were found in liver and spleen after intravenously injection for 12 weeks [29]. However, there was no damage on liver and spleen, while the injection of CdSe QDs caused apparently injuries to the liver of mice [26]. Thus, we considered that the composition and surface chemistry of nanoparticles played an important role on affecting the biological toxicity.

\section{The toxic effect on maternal reproductive function}

Ovary is the most important reproductive organs of mammals which controls the female hormones and the growth of eggs. The abnormality of ovary may influence the secretion of hormones and further affect the growth of placenta. So the ovary coefficient is important for assessing the reproductive toxic effect of Pdots. Pu et al. reported that the exposure of 15 and $45 \mathrm{mg} / \mathrm{Kg} \mathrm{Ni}$ nanoparticle to female rat apparently caused the increasing of luteinizing hormone (LH) and follicle stimulating hormone (FSH) and lowered the estradiol (E2) serum levels [30]. In addition, $\mathrm{Xu}$ et al. found that intravenously injecting CdSe/ZnS QDs into female mice caused apparent reducing of oocyte maturation rate [18]. In this study, the average ovary index of $25.0 \mu \mathrm{g}, 12.5 \mu \mathrm{g}$ and untreated group were $0.0541 \pm 0.0088 \%, 0.0565 \pm 0.0139 \%$ and $0.0606 \pm$ $0.0057 \%$ and showed no significant difference among 3 groups (Figure 5A), which meant that the injection of Pdots did not cause the weight change of the maternal ovary. To further evaluate the reproductive toxic effects of Pdots, the main female hormones level including estrogen and progesterone were examined using ELISA kits. Previous study had revealed the secretion of progesterone was essential for maintaining the normal development of embryo by reducing myometrial contractility during pregnancy, while the origin of estrogen level in plasma during pregnancy depended essentially on the fetal adrenal [31]. Therefore, both the progesterone and estrogen played important roles on female reproductive system including pregnancy and embryogenesis of mammals. The data in figure $5 \mathrm{~B}$ showed the average progesterone levels of $25.0 \mu \mathrm{g}, 12.5 \mu \mathrm{g}$ and untreated group were $1406.6 \pm 88.6 \mathrm{ng} / \mathrm{mL}, \quad 1716.6 \pm 161.3$ $\mathrm{ng} / \mathrm{mL}$, and $1788.1 \pm 70.1 \mathrm{ng} / \mathrm{mL}$ respectively, while the estrogen level (Figure 5C) of $25.0 \mu \mathrm{g}, 12.5 \mu \mathrm{g}$ and 
untreated group were $225.89 \pm 11.89 \mathrm{ng} / \mathrm{mL}, 233.86 \pm$ $8.41 \mathrm{ng} / \mathrm{mL}$, and $243.7 \pm 10.53 \mathrm{ng} / \mathrm{mL}$, respectively. Both these results indicated that Pdots did not significantly alter the female's hormones levels when compared with those of the untreated group during the first two week pregnant period under our dosages.
A

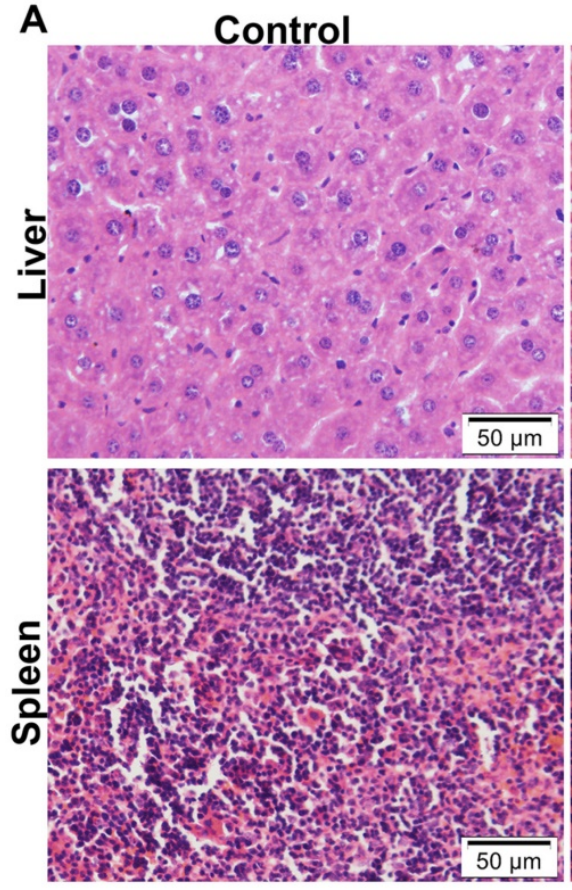

B

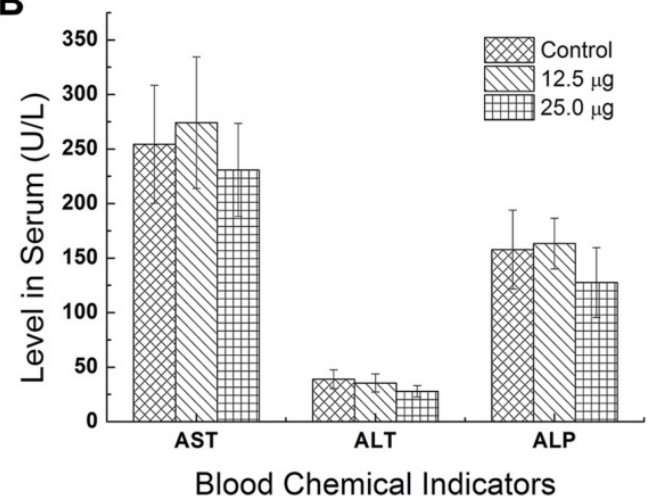

$12.5 \mu \mathrm{g}$
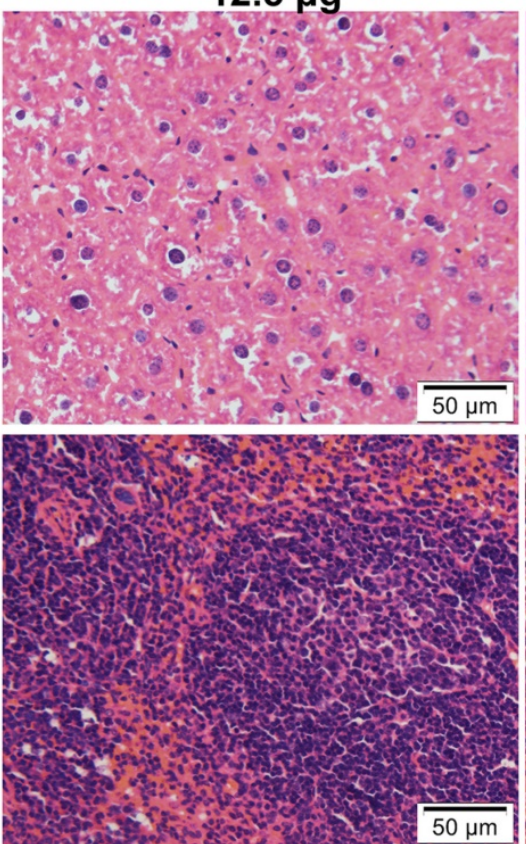

C

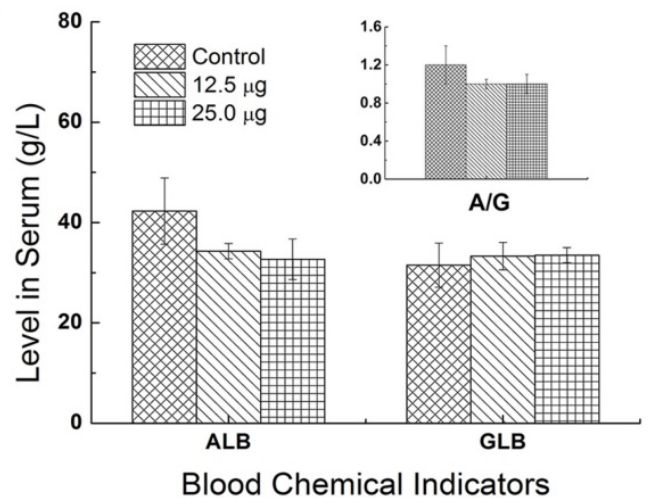

Figure 4. A) Histopathologic images of liver and spleen of maternal mice. The purple dots represented the nucleus of cells while the shallow pink dot represented the cytoplasm and intercellular substance. $B$ ) Levels of $A S T, A L T, A L P$ in serum of maternal mice, $n=6$. C) Levels of $A L B, G L B, A / G=A L B / G L B$ in serum of maternal mice, $n=6$.
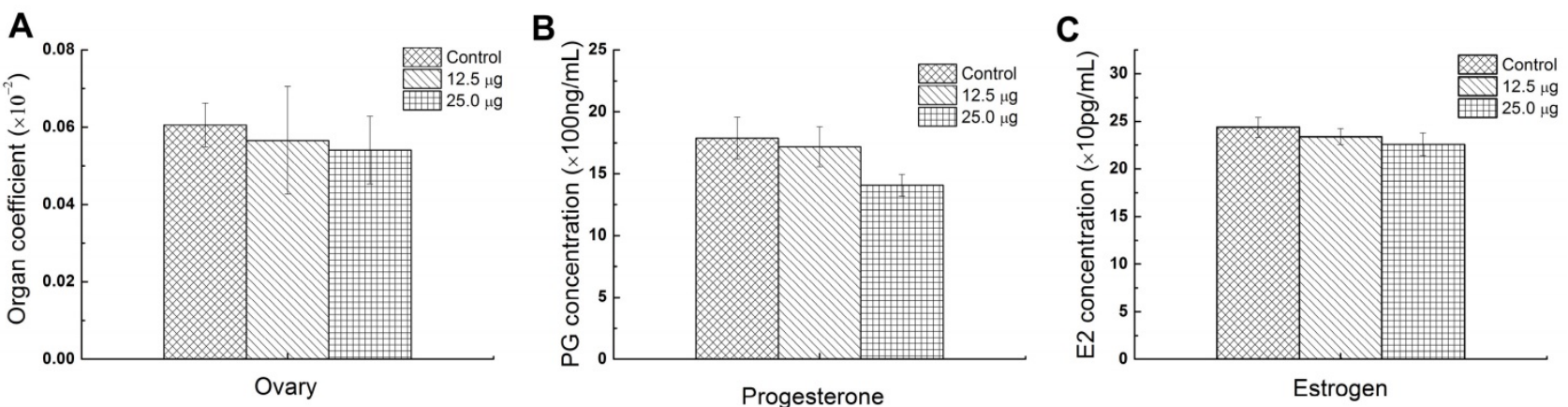

Figure 5. Reproductive function parameters of maternal mice. A) Ovary index of maternal mice, (ovary index=wet ovary weight/ maternal weight $\times 100 \%)$, $n=6$. B) Progesterone level in serum of maternal mice in ED15, $n=6$. C) Estrogen level in serum of maternal mice in $E D 15, n=6$. 


\section{The fetal toxicity of Pdots}

There were some reports about the reproductive toxicity of Cd-containing QDs and other inorganic nanoparticles. Chu et al. found that $\mathrm{Cd}^{2+}$ of Cd-containing QDs could cross through placenta barrier and dose dependently increased the fetal death rate [9]. Exposure of titanium dioxide nanoparticles during the placental period caused the pregnancy complication of maternal mouse [32] and altered the brain development related gene expression in fetus [33]. Our previous work discovered that intravenously exposure of CdSe/ZnS QDs to female mice caused the reducing of oocyte maturation rate [18]. For Pdots, there was still rare in vivo toxicity study in this part. To detect the reproductive toxicity of Pdots, we examined the effects of Pdots on the development of fetus and placenta function. In this study, the embryo including uteri, fetuses and placentas were harvested on ED15 as shown in Figure 6A, the morphologic images of fetus shown in Figure $6 \mathrm{~B}$ indicated that the limb features of fetus were normally developed in all the groups. The data shown in Table 1 indicated that the embryonic coefficients of treated groups are as normal as those of untreated group, which illustrated that Pdots had no toxic effects on the development of fetus during the early pregnant period under our exposure condition.

Table 1. Embryonic coefficients including live birth, stillbirth as well as absorption rate were evaluated and the data of average fetal weights were measured (Maternal mice of each group, $n=6$ ).

\begin{tabular}{llllll}
\hline & $\begin{array}{l}\text { Number } \\
\text { (n) }\end{array}$ & $\begin{array}{l}\text { Live birth Stillbirth } \\
\text { Rate (\%) }\end{array}$ & $\begin{array}{l}\text { Absorbed AVE Fetus } \\
\text { Rate (\%) }\end{array}$ & Rate (\%) & Weight (g) \\
\hline Control & 99 & $88.89 \%$ & $1.01 \%$ & $10.10 \%$ & $0.5402 \pm 0.0716$ \\
$12.5 \mu \mathrm{g}$ & 100 & $92.00 \%$ & $0.00 \%$ & $8.00 \%$ & $0.5779 \pm 0.0483$ \\
$25.0 \mu \mathrm{g}$ & 97 & $87.63 \%$ & $1.03 \%$ & $11.34 \%$ & $0.5493 \pm 0.0493$ \\
\hline
\end{tabular}

\section{Influence on placental development of Pdots}

Placenta is an essential organ between maternal mouse and fetus whether in transporting nutrients or protecting the fetus from external damages since there is a placenta barrier in it. Previous study indicated that, the Cd-containing QDs with different size were found in placenta. Chu et al. found that although the CdTe QDs were coated with different materials and showed different diameters (from 2 5nm), QD with smaller size was associated with the more accumulation of $\mathrm{Cd}$ in fetus, which increased the fetus death rate [9]. Zhang et al. illustrated that $\mathrm{Cd}$ contained in gestational tissue including placenta and uterus of treated groups showed significant increase compared with that of untreated group while the positive control group treated with $\mathrm{CdCl}$ only showed slight increase, which indicated that the nanoparticles, but not $\mathrm{Cd}^{2+}$ could easily penetrate the placenta and uterus [22]. The $35 \mathrm{~nm} \quad \mathrm{SiO}_{2}$ and $70 \mathrm{~nm} \quad \mathrm{TiO}_{2}$ nanoparticles caused placenta complication because of the low activation of complement and the oxidative stress in placenta [32]. All these nanomaterials caused toxicity for pregnant mice or fetus because of different mechanisms. Another interesting result was that some Pdots were found in the amniotic fluid. A few of Pdots could also be found in placenta tissues (Figure 7A, a4-a6) but no Pdots could be found in the tissue of the fetus in the treated groups (data no shown). Moreover, the average placenta weights of treated groups showed no significant difference from untreated group (shown in Figure 7B). It had been reported that placenta was originally developed at 3.5 embryonic days (ED3.5) and maturely developed at ED14.5. At the time window of ED10.5, the structure of placenta was preliminary developed well and the placenta barrier started to play its role [34, 35]. The mechanisms of placenta crossing included passive diffusion, facilitated diffusion, paracellular entry, water-fill transplacental channels and endocytosis [36]. In this study, the pathological images of placenta tissue showed that Pdots did not damage the structure of placenta (Figure 7A, a7-a9). To further investigate the pathway of Pdots, we measured the gene expression level of placenta growth factor (PGF) and vascular endothelial growth factor (VEGF) with reverse transcription-polymerase chain reaction (RT-PCR). Both VEGF and PGF play important roles in angiogenesis and regulation of vasculogenesis during the development of placenta [37]. In our study, the mRNA levels of PGF expressed in Figure 7C were $1.0081 \pm 0.1311,1.1451 \pm 0.3576$ and $1.0724 \pm 0.3348$ respectively corresponding to $25 \mu \mathrm{g}, 12.5 \mu \mathrm{g}$ and untreated group. In addition, the VEGF expression level of $25 \mu \mathrm{g}, 12.5 \mu \mathrm{g}$ and untreated group were $0.8706 \pm 0.0453,0.7800 \pm 0.1583$ and $0.9093 \pm 0.0602$ respectively. Both the results showed no obvious difference among the 3 groups. Since Pdots were found in amniotic fluid, we speculated that the existence of Pdots in amniotic fluid from two pathways: some of Pdots had entered the embryo tissue and distributed in amniotic fluid through blood circulation on the earlier embryonic stage, others of Pdots may traverse the placenta barrier through the channels mentioned above after E10.5. The reason that the Pdots existed in amniotic fluid may ascribe to the nanoparticle surface chemistry and the stage of embryonic/placental maturation [38]. 

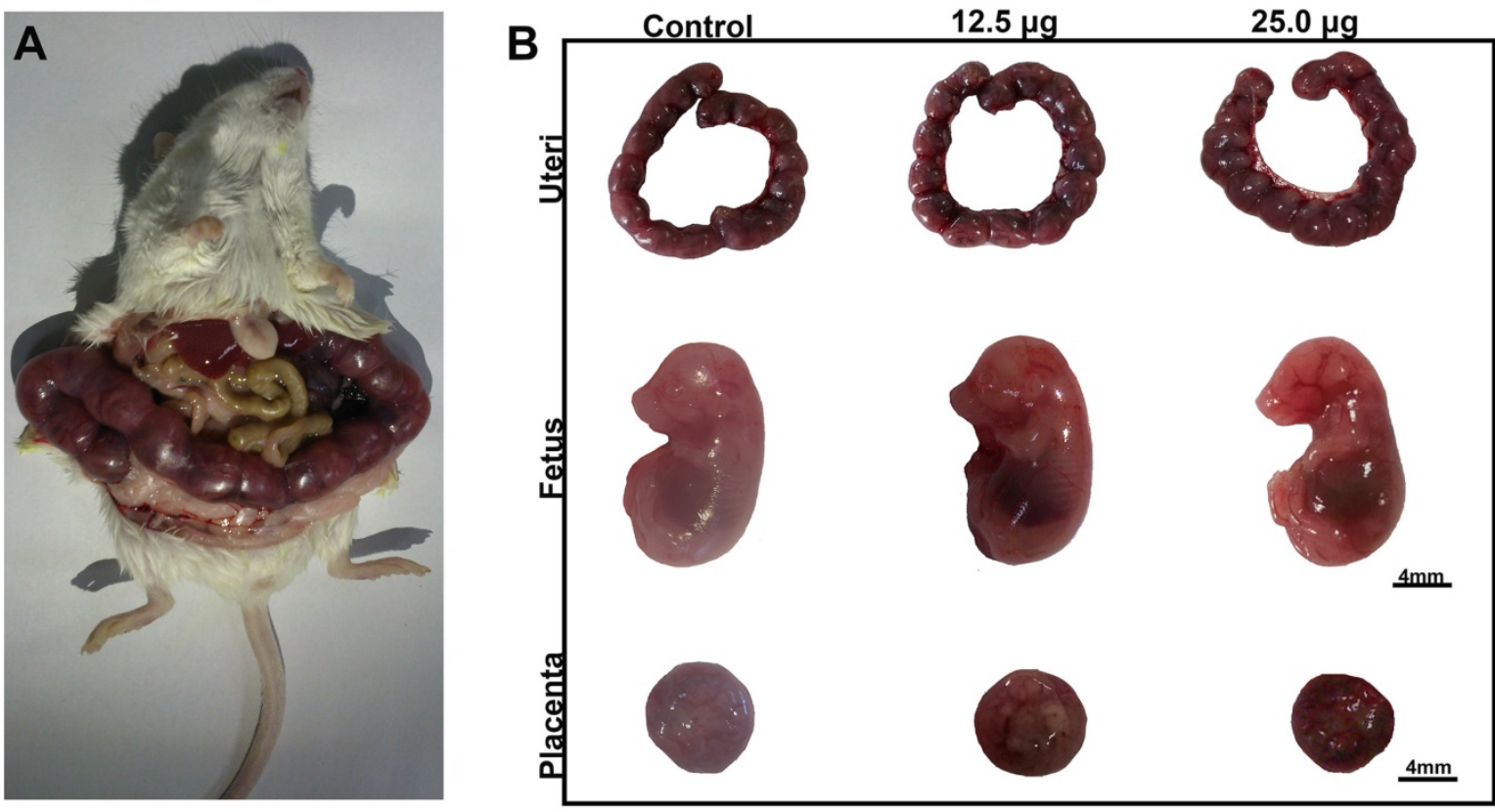

Figure 6. A) Morphological images of maternal mouse on the ED15. B) Uteri, Morphological images of fetus and placenta on ED15, scale bar: $4 \mathrm{~mm}$.
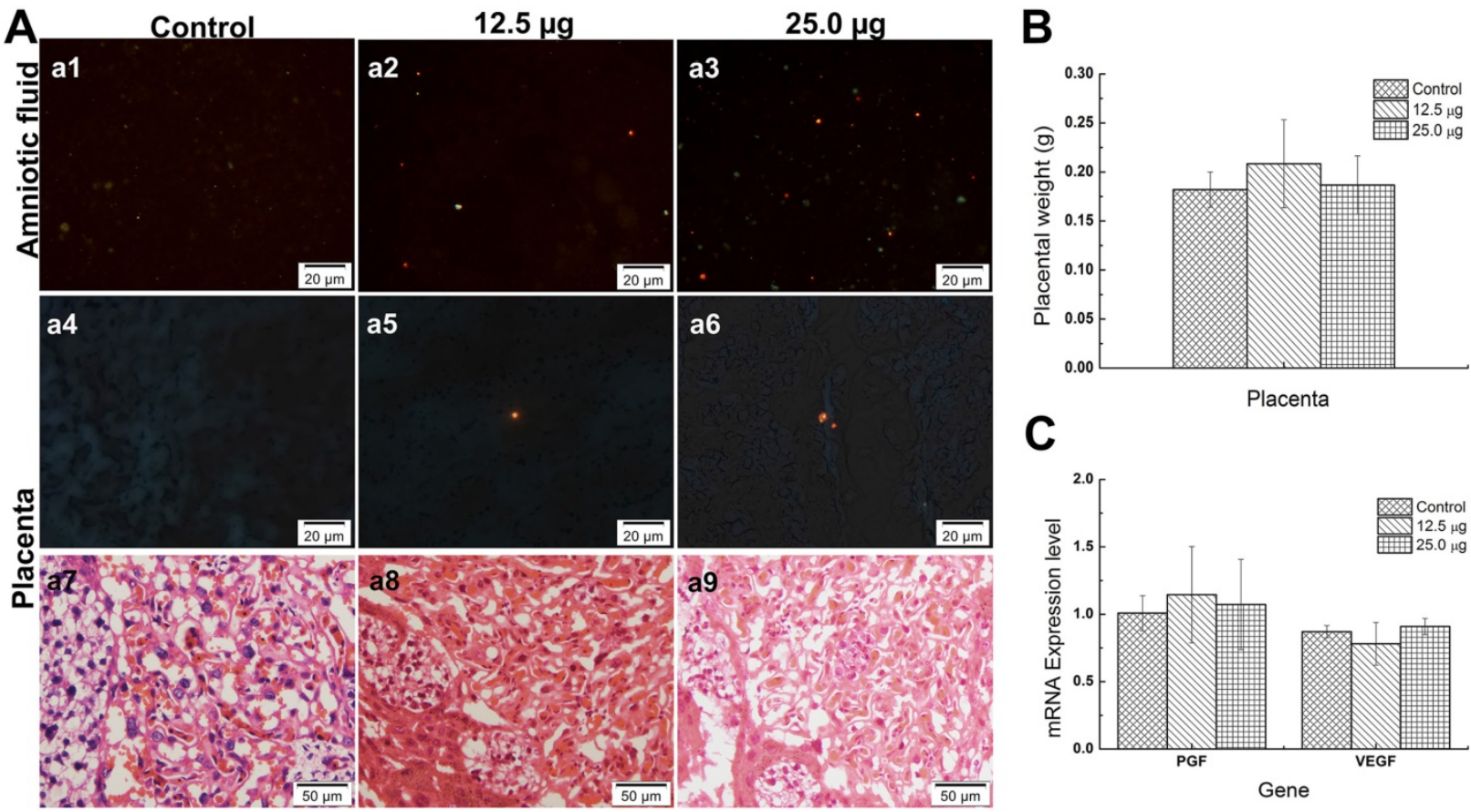

Figure 7. A) Pdots distribution in amniotic fluid of embryo (al-a3), Pdots distribution in placenta (a4-a6) and pathological section images of placenta (a7-a9). The orange-red fluorescence light dots are the aggregated Pdots in al-a6, the existences of Pdots in amniotic fluid sediment are dose dependently while only sporadic Pdots distributed in the placenta tissue. B) The average placenta weight of each group, the specimen number of $25.0 \mu \mathrm{g}, 12.5 \mu \mathrm{g}$ and untreated group were 97,100 , 99, respectively. C) The mRNA levels of placental barrier related factors and placenta growth factor, $n=6$.

\section{Conclusion}

In this study, we examined the reproductive toxicity of Pdots since there were a few studies of polymer dots on this area. The pregnant mice were exposed to Pdots with various concentrations for two weeks. Later the reproductive toxicity was evaluated from the aspects of maternal physical conditions and the development of fetus. During the process of the whole experiment, the pregnant mice did not have unusual response and strange behavior. We found that the tail intravenously injected Pdots could distribute throughout the whole maternal body through blood, even some of Pdots may pass through 
the placenta barrier. At the dosages of $25.0 \mu \mathrm{g}$ and 12.5 $\mu \mathrm{g}$, most of the Pdots gathered in maternal liver and spleen which verifies that the in vivo distribution of quantum dots does not only relate to Pdots size and surface chemistry, but also to the reticuloendothelial system in liver and spleen [39].The levels of female hormones, progesterone and estrogen, have not been interfered under the design dosage of this study either, which indicated that Pdots produced no toxic effect on female hormones under our dosages. That means the continuous exposure of Pdots have negligible impacts on pregnant mice. It is notable that, there are also some Pdots that stayed in amniotic fluid while most of the fetus could grow and develop as normal as that of the untreated group, which illustrated that Pdots have a much lower feto-toxicity than that of $\mathrm{Cd}$ containing quantum dots [9], silica and titanium dioxide nanoparticles [33]. These data demonstrated that a total dose lower than 175.0 $\mu \mathrm{g} /$ mouse of Pdots was safe for the female reproductive system of mice. These results provide some valuable and precious information for the design and development of Pdots for biomedical applications, such as living diagnostic as well as tumor targeting treatment. However, the biggest obstacle on the application of Pdots is the difficulty of quantifying its amount in vivo since it does not contain any specific element like heavy metal. We think that if the quantification and pharmacokinetic of this kind of Pdots could be solved, their potentially excellent biomedical application prospect is expectable. Thus, it is necessary to carry out more studies on the obstacles as mentioned above in the future work.

\section{Supplementary Material}

Figure S1 and Table S1.

http://www.ntno.org/v01p0261s1.pdf

\section{Acknowledgements}

This research was supported by National Basic Research Program of China (2015CB352005), National Natural Science Foundation of China (31671491 \& 61235012 \& 61335001 \& 81301318), Guangdong Natural Science Foundation (2014A030312008), China Scholarship Council (CSC) and Chinese Postdoctoral Science Foundation (2016M602523).

\section{Competing Interests}

The authors have declared that no competing interest exists.

\section{References}

1. Dabbousi BO, Rodriguez J, Mikulec FV, et al. (CdSe)ZnS Core-Shell Quantum Dot Synthesis and Characterization of a Size Series of Highly Luminescent Nanocrystallites. J Phys Chem B 1997; 1997: 9463-75.
2. Shao L, Gao Y, Yan F. Semiconductor quantum dots for biomedicial applications. Sensors (Basel). 2011; 11: 11736-51.

3. Joo KI, Fang Y, Liu Y, Xiao L, Gu Z, Tai A, et al. Enhanced Real-Time Monitoring of Adeno-Associated Virus Trafficking by Virus-Quantum Dots Conjugates. ACS Nano. 2011; 5: 3523-35.

4. Hu K, Wang H, Tang G, Huang T, Tang X, Liang X, et al. In Vivo Cancer Dual-Targeting and Dual-Modality Imaging with Functionalized Quantum Dots. J Nucl Med. 2015; 56: 1278-84

5. Soenen SJ, Montenegro JM, Abdelmonem AM, Manshian BB, Doak SH, Parak WJ, et al. The effect of nanoparticle degradation on amphiphilic polymer-coated quantum dot toxicity: the importance of particle functionality assessment in toxicology [corrected]. Acta Biomater. 2014; 10: 732-41.

6. Zhao YL, Wang X, Wu QL, Li YP, Wang DY. Translocation and neurotoxicity of CdTe quantum dots in RMEs motor neurons in nematode Caenorhabditis elegans. J Hazard Mater. 2015; 283: 480-9.

7. Yong KT, Swihart MT. In vivo toxicity of quantum dots: no cause for concern?. Nanomedicine. 2012; 7: 1641-3.

8. Li X, Yang X, Yuwen L, Yang W, Weng L, Teng Z, et al. Evaluation of toxic effects of CdTe quantum dots on the reproductive system in adult male mice. Biomaterials. 2016; 96: 24-32.

9. Chu M, Wu Q, Yang H, Yuan R, Hou S, Yang Y, et al. Transfer of quantum dots from pregnant mice to pups across the placental barrier. Small. 2010; 6: 670-8.

10. Wang X, Tian J, Yong KT, Zhu X, Lin MC, Jiang W, et al. Immunotoxicity assessment of CdSe/ZnS quantum dots in macrophages, lymphocytes and BALB/c mice. J Nanobiotechnology. 2016; 14: 10.

11. Wu C, Chiu DT. Highly fluorescent semiconducting polymer dots for biology and medicine. Angew Chem Int Ed Engl. 2013; 52: 3086-109.

12. Wu C, Hansen SJ, Hou Q, Yu J, Zeigler M, Jin Y, et al. Design of highly emissive polymer dot bioconjugates for in vivo tumor targeting. Angew $\mathrm{Chem}$ Int Ed Engl. 2011; 50: 3430-4.

13. Liu HY, Wu PJ, Kuo SY, Chen CP, Chang EH, Wu CY, et al Quinoxaline-Based Polymer Dots with Ultrabright Red to Near-Infrared Fluorescence for In Vivo Biological Imaging. J Am Chem Soc. 2015; 137: 10420-9.

14. Liu J, Li K, Liu B. Far-Red/Near-Infrared Conjugated Polymer Nanoparticles for Long-Term In Situ Monitoring of Liver Tumor Growth. Advanced Science. 2015; 2: 1500008 .

15. Ding D, Liu J, Feng G, Li K, Hu Y, Liu B. Bright far-red/near-infrared conjugated polymer nanoparticles for in vivo bioimaging. Small. 2013; 9: 3093-102.

16. Wu C, Schneider T, Zeigler M, Yu J, Schiro PG, Burnham DR, et al. Bioconjugation of Ultrabright Semiconducting Polymer Dots. J Am Chem Soc. 2010; 2010: 15410-7.

17. Saunders M. Transplacental transport of nanomaterials. Wires Nanomed Nanobi. 2009; 1: 671-84.

18. Xu G, Lin G, Lin S, Wu N, Deng Y, Feng G, et al. The Reproductive Toxicity of $\mathrm{CdSe} / \mathrm{ZnS}$ Quantum Dots on the in vivo Ovarian Function and in vitro Fertilization. Scientific reports. 2016; 6: 37677.

19. Su Y, Peng F, Jiang $Z$, Zhong $Y$, Lu Y, Jiang $X$, et al In vivo distribution, pharmacokinetics, and toxicity of aqueous synthesized cadmium-containing quantum dots. Biomaterials. 2011; 32: 5855-62.

20. Andersen H, Larsen S, Spliid H, Christensen ND. Multivariate statistical analysis of organ weights in toxicity studies. Toxicology. 1999; 136: 67-77.

21. Shirley E.A, Newnham P. The choice between analysis of variance and analysis of covariance with special reference to the analysis of organ weights in toxicology studies. Statistic in Medicine. 1984; 3: 85-91.

22. Zhang W, Yang L, Kuang H, Yang P, Aguilar ZP, Wang A, et al. Acute toxicity of quantum dots on late pregnancy mice: Effects of nanoscale size and surface coating. J Hazard Mater. 2016; 318: 61-9.

23. Sharifi S, Behzadi S, Laurent S, Forrest ML, Stroeve P, Mahmoudi M. Toxicity of nanomaterials. Chem Soc Rev. 2012; 41: 2323-43.

24. Schipper ML, Iyer G, Koh AL, Cheng Z, Ebenstein Y, Aharoni A, et al. Particle size, surface coating, and PEGylation influence the biodistribution of quantum dots in living mice. Small. 2009; 5: 126-34.

25. Tang Y, Han S, Liu H, Chen X, Huang L, Li X, et al. The role of surface chemistry in determining in vivo biodistribution and toxicity of CdSe/ZnS core-shell quantum dots. Biomaterials. 2013; 34: 8741-55.

26. Liu W, Zhang S, Wang L, Qu C, Zhang C, Hong L, et al. CdSe quantum dot (QD)-induced morphological and functional impairments to liver in mice. PLoS One. 2011; 6: e24406.

27. Fischer HC, Liu L, Pang KS, Chan WCW. Pharmacokinetics of Nanoscale Quantum Dots: In Vivo Distribution, Sequestration, and Clearance in the Rat. Advanced Functional Materials. 2006; 16: 1299-305.

28. Choi HS, Liu W, Misra P, Tanaka E, Zimmer JP, Itty Ipe B, et al. Renal clearance of quantum dots. Nat Biotechnol. 2007; 25: 1165-70.

29. Lin G, Ouyang Q, Hu R, Ding Z, Tian J, Yin F, et al. In vivo toxicity assessment of non-cadmium quantum dots in BALB/c mice. Nanomedicine. 2015; 11: 341-50.

30. Kong L, Tang M, Zhang T, Wang D, Hu K, Lu W, et al. Nickel nanoparticles exposure and reproductive toxicity in healthy adult rats. International journal of molecular sciences. 2014; 15: 21253-69.

31. Morel Y, Roucher F, Plotton I, Goursaud C, Tardy V, Mallet D. Evolution of steroids during pregnancy: Maternal, placental and fetal synthesis. Ann Endocrinol (Paris). 2016; 77: 82-9. 
32. Yamashita K, Yoshioka Y, Higashisaka K, Mimura K, Morishita Y, Nozaki M, et al. Silica and titanium dioxide nanoparticles cause pregnancy complications in mice. Nature nanotechnology. 2011; 6: 321-8.

33. Shimizu M, Tainaka H, Oba T, Mizuo K, Umezawa M, Takeda K. Maternal exposure to nanoparticulate titanium dioxide during the prenatal period alters gene expression related to brain development in the mouse. Particle and fibre toxicology. 2009; 6: 20

34 Watson ED, Cross JC. Development of structures and transport functions in the mouse placenta. Physiology. 2005; 20: 180-93.

35. Cross J, Werb Z, Fisher S. Implantation and the placenta: Key pieces of the development puzzle. Science. 1994; 266: 1508.

36. Menezes V, Malek A, Keelan1 JA. Nanoparticulate drug delivery in pregnancy placental passage and fetal exposure. Current Pharmaceutical Biotechnology. $2011 ; 12$.

37 Kurtoglu E, Altunkaynak BZ, Aydin I, Ozdemir AZ, Altun G, Kokcu A, et al. Role of vascular endothelial growth factor and placental growth factor expression on placenta structure in pre-eclamptic pregnancy. J Obstet Gynaecol Res. 2015; 41: 1533-40.

38. Yang H, Sun C, Fan Z, Tian X, Yan L, Du L, et al. Effects of gestational age and surface modification on materno-fetal transfer of nanoparticles in murine pregnancy. Scientific reports. 2012; 2: 847.

39. Hauck TS, Anderson RE, Fischer HC, Newbigging S, Chan WC. In vivo quantum-dot toxicity assessment. Small. 2010; 6: 138-44. 